\title{
RESEARCH
}

Open Access

\section{Systemic exosomal miR-193b-3p delivery attenuates neuroinflammation in early brain injury after subarachnoid hemorrhage in mice}

Niansheng Lai ${ }^{1,2,3 \dagger}$, Degang Wu ${ }^{1,2,3 \dagger}$, Tianyu Liang ${ }^{1}$, Pengjie Pan ${ }^{1}$, Guiqiang Yuan ${ }^{1}$, Xiang Li ${ }^{1}$, Haiying Li ${ }^{1}$, Haitao Shen ${ }^{1}$, Zhong Wang ${ }^{1 *}$ and Gang Chen ${ }^{1 *}$

\begin{abstract}
Background: Inflammation is a potential crucial factor in the pathogenesis of subarachnoid hemorrhage (SAH). Circulating microRNAs (miRNAs) are involved in the regulation of diverse aspects of neuronal dysfunction. The therapeutic potential of miRNAs has been demonstrated in several CNS disorders and is thought to involve modulation of neuroinflammation. Here, we found that peripherally injected modified exosomes (Exos) delivered miRNAs to the brains of mice with SAH and that the potential mechanism was regulated by regulation of neuroinflammation.
\end{abstract}

Methods: Next-generation sequencing (NGS) and qRT-PCR were used to define the global miRNA profile of plasma exosomes in aSAH patients and healthy controls. We peripherally injected RVG/Exos/miR-193b-3p to achieve delivery of miR-193b-3p to the brain of mice with SAH. The effects of miR-193b-3p on SAH were assayed using a neurological score, brain water content, blood-brain barrier (BBB) injury, and Fluoro-Jade C (FJC) staining. Western blotting analysis, enzyme-linked immunosorbent assay (ELISA), and qRT-PCR were used to measure various proteins and mRNA levels.

Results: NGS and qRT-PCR revealed that four circulating exosomal miRNAs were differentially expressed. RVG/Exos exhibited improved targeting to the brains of SAH mice. MiR-193b-3p suppressed the expression and activity of HDAC3, upregulating the acetylation of NF-KB p65. Finally, miR-193b-3p treatment mitigated the neurological behavioral impairment, brain edema, BBB injury, and neurodegeneration induced by SAH, and reduced inflammatory cytokine expression in the brains of mice after SAH.

Conclusions: Exos/miR-193b-3p treatment attenuated the inflammatory response by acetylation of the NF-kB p65 via suppressed expression and activity of HDAC3. These effects alleviated neurobehavioral impairments and neuroinflammation following SAH.

Keywords: Subarachnoid hemorrhage, Neuroinflammation, Exosomes, Brain delivery

\footnotetext{
*Correspondence: Ihy1015@suda.edu.cn; nju_neurosurgery@163.com

${ }^{\dagger}$ Niansheng Lai and Degang Wu contributed equally to this work.

${ }^{1}$ Department of Neurosurgery \& Brain and Nerve Research Laboratory, The

First Affiliated Hospital of Soochow University, 188 Shizi Street, Suzhou

215006, Jiangsu Province, China

Full list of author information is available at the end of the article
}

(c) The Author(s). 2020 Open Access This article is distributed under the terms of the Creative Commons Attribution 4.0 International License (http://creativecommons.org/licenses/by/4.0/), which permits unrestricted use, distribution, and reproduction in any medium, provided you give appropriate credit to the original author(s) and the source, provide a link to the Creative Commons license, and indicate if changes were made. The Creative Commons Public Domain Dedication waiver (http://creativecommons.org/publicdomain/zero/1.0/) applies to the data made available in this article, unless otherwise stated. 


\section{Background}

Aneurysmal subarachnoid hemorrhage (SAH) typically results from a ruptured aneurysm. It is a clinical syndrome associated with mortality of $45 \%$ and morbidity of approximately 6-16 per 100,000 individuals annually worldwide, often in young individuals. SAH accounts for $5-7 \%$ of the total incidence of stroke [1, 2]. A major prognostic determinant is the character of the initial hemorrhage that causes early brain injury (EBI) and early cerebral vasospasms (CVS) and may be associated with delayed cerebral ischemia (DCI) [3]. Several lines of evidence suggest that EBI after SAH may be the leading factor contributing to unfavorable outcomes following SAH $[4,5]$. The evidence suggests that several pathophysiological disorders during EBI activate inflammatory cascades [6]. It is of paramount importance to develop novel neuroprotective therapies to enhance recovery in the period of EBI after SAH.

MicroRNAs (miRNAs) are a family of non-coding RNAs of 17-24 nucleotides that regulate the expression of several target genes at the post-transcriptional level [7-11]. Previous studies demonstrated that miRNAs were involved in numerous physiological/pathological processes and were particularly appealing diagnostic and therapeutic tools for various diseases, including stroke, Parkinson's disease, traumatic brain injury (TBI), and Alzheimer's disease. Despite the fact that expression levels of miRNAs have measured in cerebrospinal fluid and in circulation, there are few studies investigating changes of miRNAs after SAH [12-14]. The blood-brain barrier (BBB) has been proven to be a major obstacle for delivery of drugs to the brain. It has been estimated that $98 \%$ of all potent molecules have no clinical effect because of their inability to cross the BBB $[11,15]$.

Several lines of evidence suggested that exosomes, which are lipid membrane vesicles of 30 to $100 \mathrm{~nm}$ in diameter, cross the BBB and specialize in long-distance intercellular communication, facilitating transfer of proteins, lipids, functional mRNAs, and microRNAs for subsequent protein expression in target cells [16]. Exosomes released by brain cells are able to cross the BBB and can be detected in the systemic circulation. Similarly, endothelial and peripheral cells secrete exosomes into the circulation. Exosomes can be enriched from peripheral blood samples and can be used to detect various proteins, lipids, and nucleic acids [17]. Exosomes have been used as drug delivery vehicles for the treatment of several CNS diseases $[11,18-21]$. Exosomes are currently under investigation as therapeutic options for stroke [22]. Rabies virus glycoprotein (RVG) has been engineered for the exosomal surface using fused protein lysosome-associated membrane glycoprotein $2 \mathrm{~b}$ (Lamp2b)-RVG, a preferred option to address this problem, because they cross the BBB and transport miRNAs specifically into the CNS [11, 23]. Unlike intracerebroventricular injections, RVG/exosomes (RVG/ Exos) are injected intravenously and are thereby easily used for noninvasive treatment of CNS diseases. They are capable of transporting small molecules and macromolecular drugs rapidly to the CNS within the liver or degradation by mononuclear phagocyte system.

To the best of our knowledge, our study is the first of exosome-based miRNA therapy in SAH. We measured expression profiles of plasma exosomal miRNAs in patients with SAH using exosomal miRNA sequencing and qRTPCR. Then, we tested whether exosomes derived from bone marrow mesenchymal stem cells (BMSCs) would transfer differentially expressed plasma exosomal miRNAs to brain tissues, leading to upregulation of miRNAs in brain tissues in mice after SAH. We evaluated the possibility that Exos/ miRNAs inhibited neuroinflammation and exhibited neuroprotective effects against EBI in SAH.

\section{Methods}

\section{Ethics and animals}

Study participants were recruited from the Department of Neurosurgery at The First Affiliated Hospital of Wannan Medical College, Wuhu, China. The study was performed in accordance with the Declaration of Helsinki. Written informed consent was received from participants or valid proxies (family or a professional not directly involved in the study). All experiments were approved by the Ethics Committee of the First Affiliated Hospital of Soochow University and Research Ethics Committee of Wannan Medical College, and were performed according to the guidelines of the National Institutes of Health on the care and use of animals. All adult male C57BL/6 mice weighing 25-30g were purchased from the Animal Center of the Chinese Academy of Sciences, Shanghai, China. All mice were housed in temperature- and humidity-controlled animal quarters with a 12-h light/dark cycle. Every effort was made to minimize the number of animals used and their suffering.

\section{SAH model in mice}

As described in a previous study [24], mice were intraperitoneally anesthetized with $4 \%$ chloral hydrate $(0.4 \mathrm{~g} /$ $\mathrm{kg}$ body weight). They were then placed in the stereotaxic apparatus. A specially made needle with a rounded tip and a side hole was stereotactically inserted into the suprachiasmatic cistern to produce the SAH model. The needle $\left(3.5 \mathrm{~mm}\right.$ anterior to the bregma, angle of $\left.28-30^{\circ}\right)$ was inserted in the midline until it reaches the skull (2 $\mathrm{mm}$ anterior to the chiasma, $0.5 \mathrm{~mm}$ retracted). Next, $0.1 \mathrm{ml}$ non-heparinized allologous arterial blood was slowly injected into the suprachiasmatic cistern over 20 s; $0.1 \mathrm{ml}$ saline was injected for the sham group. To prevent dehydration, the mice were injected subcutaneously with $1 \mathrm{ml}$ of $0.9 \%$ saline after surgery. We observed that the inferior basal temporal lobe was always stained with 
blood. Plasma and brain tissues surrounding the hemorrhage located in the temporal base were sampled and subjected to analysis after the mice were sacrificed at the indicated time points.

\section{Cell culture}

Bone marrow from 6-week-old adult male mice was mechanically harvested from femurs as previously described [25]. Cells were washed in PBS and suspended in DMEM supplemented with $20 \%$ fetal bovine serum and antibiotics (all from GIBCO, USA). Three days later, nonadherent cells were removed by replacing fresh medium, and cells remaining tightly adhered to the plastic flasks were considered to be $P O$ BMSCs. BMSCs were used until the eighth passage (P8) for exosome collection.

\section{Lentivirus production and infection}

The 1356-bp sequence of the exosomal membrane protein gene Lamp2b (lysosomal-associated membrane protein $2 \mathrm{~b}$ ) was fused with the targeting peptide RVG and was amplified using $293 \mathrm{~T}$ cell cDNA as a template, with the following primers: $\mathrm{m}-\mathrm{RVG}-\mathrm{lamp} 2 \mathrm{~b}-\mathrm{F}$ and $\mathrm{m}$ RVG-lamp2b-R (Table 1). EcoR I and BamH I are endonuclease sites for plasmid construction with the pHBLVCMV-MCS-3FLAG-EF1- ZsGreen-T2A-PURO vector. The sequences of all constructs were confirmed using DNA sequencing (HanBio Biotech, China). The cells $\left(2 \times 10^{5}\right.$ per well in 6-well plates) were transfected with LipoFiter $^{\text {Tw }}$ Liposomal Transfection Reagent (Hanbio Biotechnology, China), as per the protocol provided by the lentivirus manufacturer. Retroviral infection was performed as previously described [26].

\section{Isolation and characterization of exosomes}

Exosomes were purified from cell culture supernatants in serum-free medium of BMSCs. Prior to culture medium collection, BMSCs were washed twice with PBS and were cultured in serum-free medium at $37^{\circ} \mathrm{C}$ in a humidified

Table 1 Real-time PCR primers used for quantification of mRNA expression in this study

\begin{tabular}{|c|c|c|}
\hline Primer name & Sequence $\left(5^{\prime} \rightarrow 3^{\prime}\right)$ & \\
\hline \multirow[t]{2}{*}{ 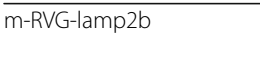 } & Forward & CACCATTTGGATGCCCGAGA \\
\hline & Reverse & CCGTTGGATGCTCTCTTCCC \\
\hline \multirow[t]{2}{*}{ Hdacl } & Forward & ACGGCATTGACGACGAATCCTATG \\
\hline & Reverse & CTGAGCCACACTGTAAGACCACTG \\
\hline \multirow[t]{2}{*}{ Hdac2 } & Forward & AGACTGGAATAGGACGGACGGATG \\
\hline & Reverse & GTCATTTACCCAAGGGCTGGCTAC \\
\hline \multirow[t]{2}{*}{ Hdac3 } & Forward & TGGAACAGGTGACATGTATGAA \\
\hline & Reverse & GAAAAGGTGCTTGTAACTCTGG \\
\hline \multirow[t]{2}{*}{ Hdac8 } & Forward & GATACTATTGCCGGAGATCCAA \\
\hline & Reverse & GATAGCGTITCCCTAGGATGA \\
\hline \multirow[t]{2}{*}{ Rela } & Forward & GCTACACAGGACCAGGAACAGTTC \\
\hline & Reverse & CTTGCTCCAGGTCTCGCTTCTTC \\
\hline \multirow[t]{2}{*}{$B C L-2$} & Forward & GATGACTTCTCTCGTCGCTAC \\
\hline & Reverse & GAACTCAAAGAAGGCCACAATC \\
\hline \multirow[t]{2}{*}{ Caspase-3 } & Forward & GAAACTCTTCATCATTCAGGCC \\
\hline & Reverse & GCGAGTGAGAATGTGCATAAAT \\
\hline \multirow[t]{2}{*}{$L-1 \beta$} & Forward & GCAGAGCACAAGCCTGTCTTCC \\
\hline & Reverse & ACCTGTCTTGGCCGAGGACTAAG \\
\hline \multirow[t]{2}{*}{ IL-6 } & Forward & ACGTAGCTAGCTAGTCGGTATG \\
\hline & Reverse & TCGTAGCTTGGCTAGTCGATCG \\
\hline \multirow[t]{2}{*}{ TNF- $a$} & Forward & ATGTCTCAGCCTCTTCTCATTC \\
\hline & Reverse & GCTTGTCACTCGAATTITGAGA \\
\hline \multirow[t]{2}{*}{$\beta$-actin } & Forward & GCTGTCCCTGTATGCCTCTG \\
\hline & Reverse & CGCTCGTTGCCAATAGTGATG \\
\hline MiRNA-193b-3p & Forward & AACTGGCCCTCAAAGTCCCGCT \\
\hline MiRNA-486-3p & Forward & gCGGGGCAGCTCAGTACAGGAT \\
\hline MiRNA-369-3p & Forward & accggccgcggAATAATACATGGTTGATCTIIT \\
\hline MiRNA-410-3p & Forward & ccgcgggAATATAACACAGATGGCCTGT \\
\hline MiRNA-136-3p & Forward & ccgcggCATCATCGTCTCAAATGAGTCT \\
\hline MiRNA-195-5p & Forward & cgccggTAGCAGCACAGAAATATTGGC \\
\hline Cel-miR-39 & Forward & cgUCACCGGGUGUAAAUCAGCUUG \\
\hline Universal reverse primers & Reverse & CAGGTCCAGTTITITIITIITITCGT \\
\hline
\end{tabular}


atmosphere of $5 \% \mathrm{CO}_{2}$ for $48 \mathrm{~h}$. The culture medium supernatants or plasma samples were collected and subjected to sequential ultracentrifugation at $2000 \mathrm{~g}$ for 10 min at $4{ }^{\circ} \mathrm{C}$ to remove cell debris and then passed through $0.22 \mu \mathrm{m}$ filters, spun at 10,000 $\mathrm{g}$ for $30 \mathrm{~min}$, then $100,000 \mathrm{~g}$ for $4 \mathrm{~h}$ at $4{ }^{\circ} \mathrm{C}$. The exosomes were washed once with PBS and resuspended for further characterization.

Exosomes were adsorbed on carbon-coated nickel grids for $1 \mathrm{~h}$, subsequently washed three times with PBS for $5 \mathrm{~min}$, and fixed with $2 \%$ formaldehyde for $10 \mathrm{~min}$. Samples were contrasted using uranyl acetate and lead citrate (Sigma-Aldrich, USA). After three washings in deionized water, grids were dried for several minutes and finally examined using a TECNAI-10 transmission electron microscope (TEM; Philips, Netherlands).

\section{MiR-193b-3p loading}

Exosomes at total protein concentration of $20 \mu \mathrm{g}$ (measured using a BCA Assay kit, Beyotime) and $20 \mu \mathrm{l}$ of miR193b-3p mimics or scrambled miRNAs (GenePharma, China) were mixed in $180 \mu \mathrm{l}$ of nucleofector buffer (Cell Line Nucleofector Kit V, Amaxa) and electroporated at $350 \mathrm{~V}$ and $150 \mu \mathrm{F}$ in Nucleofector IIs/2b device. To remove the unincorporated miRNA mimics, exosomes were washed in cold PBS $\left(4{ }^{\circ} \mathrm{C}\right)$ twice by sequential ultracentrifugation. Efficiency of transfection was validated using qRT-PCR for detection of miR-193b-3p levels.

\section{RNA isolation and qRT-PCR}

Total RNA was extracted from exosomes or brain tissues using QIAzol Lysis Reagent (Qiagen, Germany) according to the manufacturer's instructions.

For analysis of mRNA levels, reverse transcription (RT) was performed using a Fast Quant RT Kit (with gDNase; Tiangen Biotech, China), and cDNAs were used for qRTPCR using SuperReal PreMix Color (SYBR Green; Tiangen Biotech, China). The primer sequences are listed in Table 1.

For analysis of miRNA levels, total RNA was reversetranscribed to cDNA using an miRcute miRNA FirstStrand cDNA Synthesis Kit (Tiangen Biotech, China), and qRT-PCR was carried out using an miRcute miRNA qPCR Detection Kit (SYBR Green; Tiangen, Biotech, China).

All PCR reactions were run in triplicate, and mRNA or miRNA levels were expressed relative to levels of $\beta$-actin, cel-miR-39, or U6 snRNA. The results of PCR reactions of mRNA and miRNA expression in tissues and medium were calculated using the $2^{-\Delta \Delta C t}$ method, and miRNA expression in plasma was calculated using the $2^{-\Delta \mathrm{Ct}}$ method.

\section{Western blot analysis}

As described previously [5], frozen samples or BMSCs cells were lysed mechanically in lysis buffer (Beyotime, China). The bicinchoninic acid (BCA) method was used to measure the concentration of brain tissues using an enhanced BCA Protein Assay kit (Beyotime, China). Molecular weight markers $(5 \mu \mathrm{l} /$ lane; Thermos Scientific, USA) and protein samples $(20 \mu \mathrm{g} / \mathrm{lane})$ were separated on $10 \%$ sodium dodecyl sulfate-polyacrylamide gels (SDS-PAGE) and electrophoretically transferred onto polyvinylidene difluoride membranes (PVDF, Millipore Corporation, USA). Then, membranes were blocked with $5 \%$ non-fat milk for $1 \mathrm{~h}$ at room temperature and then were incubated with primary antibodies in $5 \%$ BSA (diluted in PBS with $0.1 \%$ Tween 20) overnight at $4{ }^{\circ} \mathrm{C}$. The primary antibodies were as follows: goat antiLamp2b (1:1000, Abcam, USA), mouse anti- $\beta$-actin (1: 5000, Abcam, USA), mouse anti-CD63 (1:1000, Abcam, USA), rabbit anti-Alix (1:1000, Abcam, USA), rabbit anti-GM130 (1:1000, Abcam, USA), rabbit anti-HDAC3 (Histone deacetylase 3) (1:5000, Abcam, USA), rabbit anti-acetyl-NF-kB p65 (1:1000, CST, USA), and mouse anti-GAPDH (1:5000, Abcam, USA). Corresponding HRP-conjugated anti-goat, anti-rabbit, or anti-mouse (1: 10,000 , CST, USA) secondary antibodies were incubated for $2 \mathrm{~h}$ at room temperature. Bands were visualized using an enhanced chemiluminescence (ECL) kit (Affinity, China). The relative quantity of proteins was analyzed using Image J software (NIH, USA) and normalized to quantities of loading controls.

\section{Enzyme-linked immunosorbent assay}

At $24 \mathrm{~h}$ post-SAH, mice were anesthetized and brain tissues were removed. The brain tissues were mechanically homogenized in $0.9 \%$ normal saline at $200 \mathrm{mg} / \mathrm{ml}$ and centrifuged at $12,000 \mathrm{rpm}$ for $10 \mathrm{~min}$ at $4{ }^{\circ} \mathrm{C}$. The concentrations of IL-1 $\beta$, IL- 6 , and TNF- $\alpha$ in brain tissue homogenates were quantified using specific enzyme-linked immunosorbent assay (ELISA) kits for mice (Elabscience Biotechnology, China) according to the manufacturer's instructions. The final concentration of cytokines was measured using OD values.

\section{Neurological behavioral impairment}

At $24 \mathrm{~h}$ after SAH, all mice were examined for neurological behavioral impairment by an independent investigator blinded to procedure information using the Garcia test [27]. The Garcia test consists of spontaneous activity, tail suspension movement of all limbs, forelimb outstretching, climbing, touching of trunk, and vibrissae touching. Every test was scored as 0 to 3 . An 18-point scoring system was used to evaluate the sensorimotor deficits. Higher scores represent milder neurological deficits $[6,27]$.

\section{Brain edema}

At $24 \mathrm{~h}$ post-SAH, mice were anesthetized and brain tissues were removed. Samples removed from brainstem 
and cerebellum were weighed immediately (wet weight, WW), and then dried for $72 \mathrm{~h}$ at $100{ }^{\circ} \mathrm{C}$ to obtain dry weight (DW). The percentage of brain water content (BWC) was calculated as: [(wet weight - dry weight)/wet weight] $\times 100 \%$.

\section{Fluoro-Jade C staining}

Neurodegeneration was detected using Fluoro-Jade C (FJC) staining as previously described [6]. After deparaffinization and rehydration, the brain sections were successively incubated with $80 \%$ alcohol containing $1 \%$ $\mathrm{NaOH}$ for $5 \mathrm{~min}, 70 \%$ alcohol for $2 \mathrm{~min}, 0.06 \%$ potassium permanganate for $10 \mathrm{~min}$, and $0.0001 \%$ FJC (AG325, Millipore, Germany) working solution for 30 min. Next, sections were washed and dried in an incubator for $10 \mathrm{~min}$ and cleared in xylene and coverslipped with a non-aqueous, low-fluorescence, styrene-based mounting medium (Sigma-Aldrich, USA). Microscopy of the stained brain sections was performed by an experienced pathologist who was blinded to the experimental condition.

\section{Blood-brain barrier injury}

BBB permeability was described in a previous report [28]. Briefly, we injected mice with $2 \%$ Evan's blue dye ( $5 \mathrm{ml} / \mathrm{kg}$, Sigma-Aldrich) at $24 \mathrm{~h}$ post-SAH. One hour later, the mice were subjected to systemic intracardiac perfusion with PBS to clear intravascular Evan's blue dye. The mice were euthanized, with brain samples subsequently harvested and homogenized in 50\% trichloroacetic acid. Evan's blue dye in supernatants was measured at $620 \mathrm{~nm}$ using a spectrofluorophotometer. The results were expressed as micrograms of Evan's blue per gram of brain tissue.

\section{Statistical analysis}

All data were expressed as mean \pm standard deviation. Before analysis, data sets in each group were tested for normality of distribution using the Kolmogorov-Smirnov test. Differences between groups were assessed using the Mann-Whitney $U$ test and/or the two-sided unpaired Student's test. Multiple comparisons between more than two groups were made using the Kruskal-Wallis test or ANOVA. Differences were considered statistically significant when $p<0.05$. MedCalc version 13.0.0 (Broekstraat 529030, Mariakerke, Belgium) was used for all statistical analyses.

\section{Results}

Distinct plasma exosomal miRNA profiles in SAH patients MiRNA sequence expression of six plasma samples from three control individuals (two women and one man, ages 58,64 , and 60 , respectively) and three $\mathrm{SAH}$ patients (two women and one man, ages 55, 63, and 66, respectively) was analyzed to identify potential biomarkers. Plasma was taken at $24 \mathrm{~h}$ after $\mathrm{SAH}$ and was obtained in the fasting state in each healthy control. Next-generation sequencing (NGS) of these samples yielded 9 to 15 million reads aligned to the reference human genome sequence. These reads corresponded to $>50,000$ different RNA sequences. From a list of 2139 known miRNAs, only 746 were considered as being expressed, with raw read counts $\geq 1$ in at least one sample. When we analyzed the expression spectrum of individual miRNAs in SAH patients and control plasma exosome samples, we found distinct profiles of expression (Supplementary data). To further assess the differences between the groups, we performed global statistical analysis to detect truly differentially expressed miRNA sequences (adjusted $p$ value $<0.05, \mid \log 2$ (fold change $\mid>1$ and expression level not low)). NGS permitted identification of a group of plasma exosomal miRNAs that were differentially expressed in SAH patients, yielding six miRNAs that were significantly differentially expressed in SAH patients and control plasma exosome samples: hsamiR-369-3p, hsa-miR-136b-3p, hsa-miR-410-3p, hsa-miR195-5p, hsa-miR-486-3p, and hsa-miR-193b-3p (Table 2).

\section{Validation of NGS data by qRT-PCR in SAH patient cohort and $\mathrm{SAH}$ model in mice}

Expression of exosomal miRNAs from a total of 20 serum samples was analyzed, including samples from patients with SAH $(n=10)$ and samples from healthy control subjects $(n=10)$. The cohort consisted of four males and six females with a median age of 60 years (age range 55-67 years). Healthy controls consisted of four males and six females with a median age of 61 years (age range 54-66 years). We determined whether these six plasma exosomal miRNAs could serve as circulating markers by

Table 2 Selected differentially expressed miRNAs in NGS

\begin{tabular}{llll}
\hline miRNA name & $\log 2$ (fold change) & $P$ value & Expression in SAH (vs control) \\
\hline hsa-miR-369-3p & -1.17 & 0.036 & Downregulated \\
hsa-miR-136-3p & -1.06 & 0.042 & Downregulated \\
hsa-miR-410-3p & -1.55 & 0.0079 & Downregulated \\
hsa-miR-195-5p & 1.03 & 0.027 & Upregulated \\
hsa-miR-486-3p & 1.03 & 0.018 & Upregulated \\
hsa-miR-193b-3p & 1.08 & 0.018 & Upregulated \\
\hline
\end{tabular}


comparing their plasma levels between SAH patients and normal controls. qRT-PCR confirmed that four miRNAs (hsa-miR-369-3p, hsa-miR-410-3p, hsa-miR193b-3p, and hsa-miR-486-3p) showed significantly differential expression between the experimental group (24 $\mathrm{h}$ post-SAH) and healthy controls (Fig. 1a). To further confirm the significance of the observed differences in exosomal miR-193b-3p expression, the expression of plasma exosomal miR-193b-3p remained statistically significant relative to controls in a mice SAH model, whereas expression levels of miR-193b-3p were lower than controls in brain tissues of SAH mice (Fig. 1b, c).

\section{Manufacture and characterization of RVG/Lamp2b- modified exosomes}

To generate RVG/Lamp2b/Exos, BMSCs were transduced with lentiviral vectors of HBLV-RVG/Lamp2b plasmid or negative control (NC). We used western blot analysis to measure protein levels of Lamp2b in these four groups to validate whether the lentiviral vectors were successfully infected into BMSCs (Fig. 2a). Then, we purified exosomes from culture supernatants of BMSCs. To analyze the characterization of RVG/Exos derived from BMSCs, the morphology of the exosomes was observed using TEM, revealing a population with typical exosomal pellets (Fig. 2b). Western blot analysis showed that Lamp2b, CD63, and GM130 (a Golgi marker), as well as the endocytic pathway and formation-associated proteins Alix were expressed in purified exosomes (Fig. 2c). Next, we examined the efficacy of exosome-encapsulated cargoes. MiR193b-3p levels in isolated exosomes that were loaded with miR-193b-3p mimics or scrambled miRNAs via electroporation were measured using qRT-PCR analysis. We found that levels of miR-193b-3p in exosomes transfection
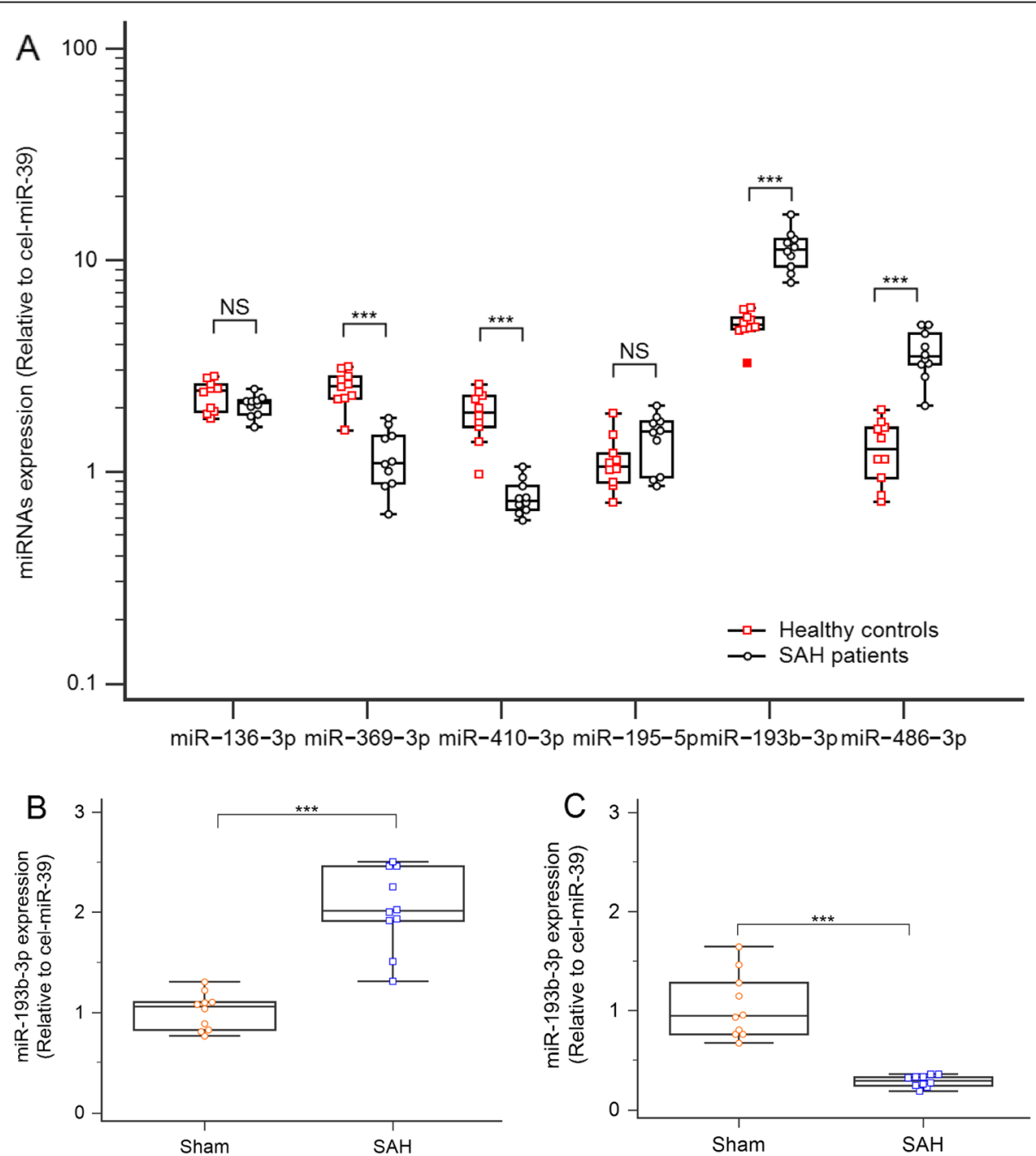

Fig. 1 Expression levels of circulating exosomal miRNAs and miRNA in the brain after SAH. a Expression profiles of exosomal miRNAs after SAH. $\mathbf{b}$ Expression of miR-193b-3p in plasma exosomes of the SAH mice and sham mice. $\mathbf{c}$ Expression of miR-193b-3p in brains of the SAH mice and sham mice (24 h later, $n=10$ ) 


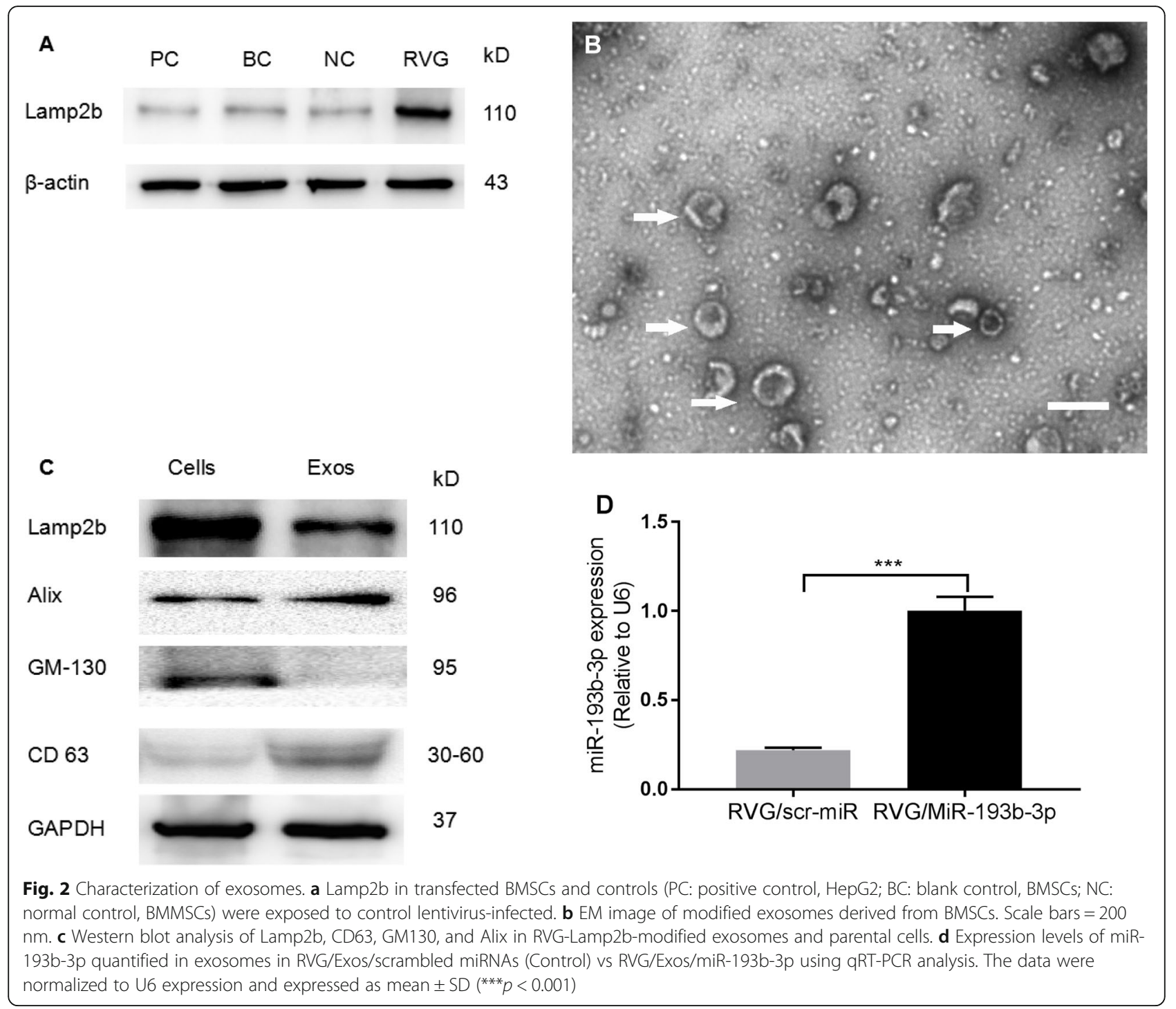

with miR-193b-3p mimics were significantly higher than in those transfected with the negative control (Fig. 2d).

\section{Engraftment of targeted RVG/Exos in the brain}

To detect the presence of the RVG/Exos in the brain, we established a SAH model in mice. Unmodified/Exos and RVG/Exos were loaded with FAM-labeled miR-193b-3p. The slides from brains in the SAH model were observed at $2 \mathrm{~h}$ after injection (Fig. 3a). We found substantially more FAM-labeled Exos in the inferior basal temporal lobe of mice in the RVG/Exos group than that in the unmodified/Exos group, and almost no FAM could be observed in the inferior basal temporal lobe of the miR193b-3p group (Fig. 3b). These data suggested that, compared with unmodified/Exos, RVG/Exos efficiently delivers miR-193b-3p into the hemorrhage region of the brain. MiRNAs were barely endocytosed by the cells independently. Another interesting finding was that FAM was localized mainly in the nucleus. These results suggested that miR-193b-3p target genes were probably localized in the nucleus (Fig. 3b).

\section{Expression patterns of histone deacetylase 3 in SAH and effects of miR-193b-3p on HDAC3 target expression}

Using miRNA target prediction algorithms (TargetScan 7.0 and miRanda 3.3a), we identified the potential targets of miR-193b-3p. We also noticed that miR-193b-3p has the potential to regulate HDAC3 expression in a previous study [29]. HDACs in human are divided into four classes. Class I (HDACs 1, 2, 3, 8) is localized mainly in the nucleus and expressed in all tissues; their highest expression is observed in the brain [30,31]. We then measured the dynamic changes and expression levels of class I HDACs in the brain of mice after SAH. We found that, in comparison with the sham group, the mRNA levels of Hdac3 reached peak levels at $12-24 \mathrm{~h}$ after $\mathrm{SAH}$, and 


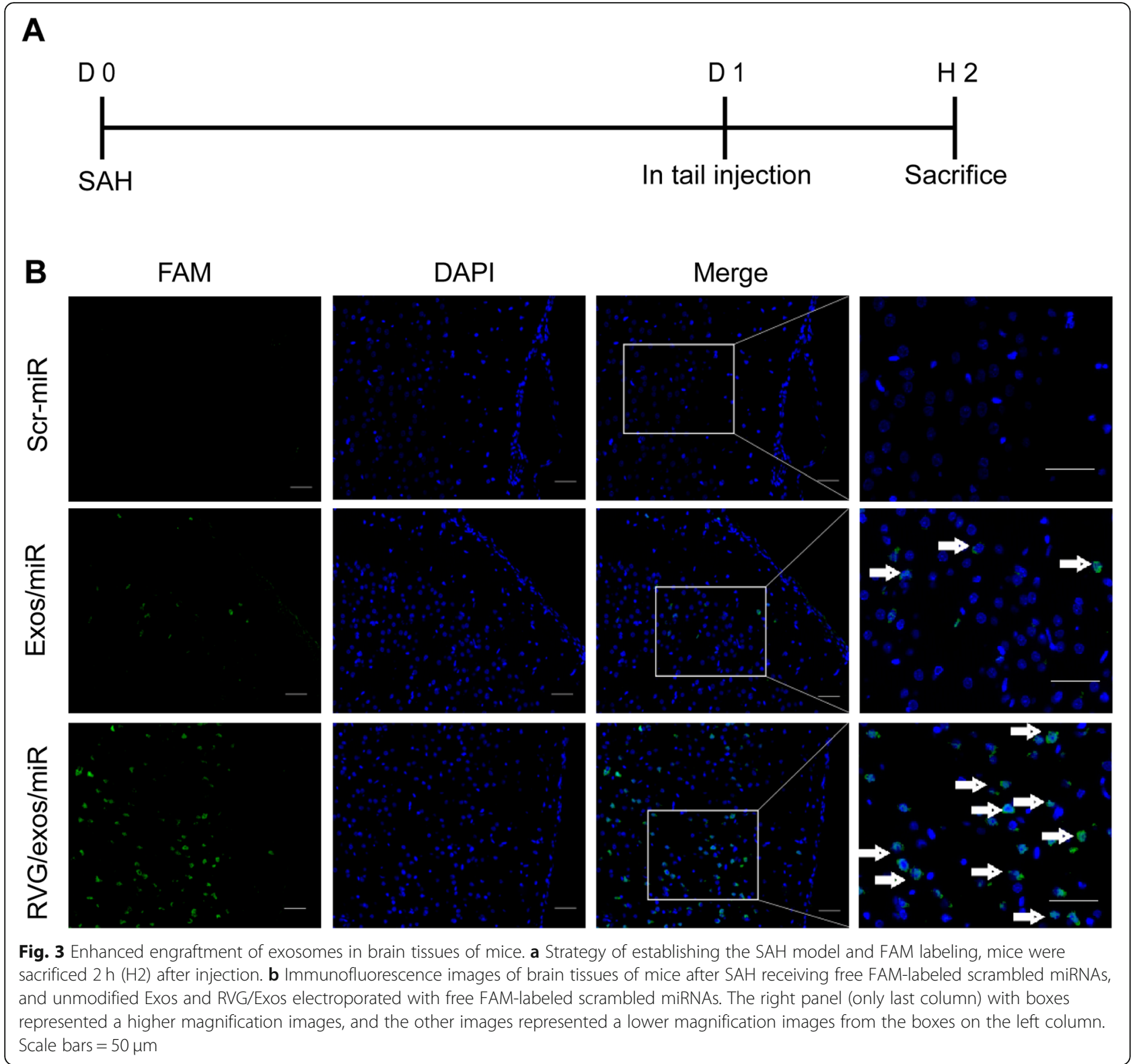

protein levels of HDAC3 reached a peak at 24 h after SAH (Fig. 4c, e). We also found that, in comparison with the sham group, expression levels of class I HDACs (HDACs 1, 2, 8) did not reach peak levels before $24 \mathrm{~h}$ after SAH (Fig. 4a, b, d). Lysine acetylation of the NF-kB p65 (Rela) has been shown to be indispensable for the NF- $\mathrm{kB}$ pathway activity regarding inflammatory mediators, playing a vital role in response to damage stimuli $[32,33]$. We found that NF-kB p65 expression levels were higher in the $\mathrm{SAH}$ group than in the sham group $(P<0.001)$. Rela mRNA levels were not significantly different after the Exos/miR-193b-3p treatment (Fig. 4g). HDAC3 levels were lower after Exos/miR-193b-3p treatment (Fig. 4f, h), whereas ac-p65 protein levels were higher in the nucleus (Fig. 4i). Our data suggest that
miR-193b-3p suppresses HDAC3 expression and activity, leading to increased ac-p65 protein levels.

Expression levels of $B C L-2$, caspase-3, IL-1 $\beta$, IL-6, and TNF$a$ in various groups

We used qRT-PCR to quantify downstream gene expression levels in the NF- $\mathrm{B}$ B pathway. Caspase-3 gene expression was elevated after SAH and lower after Exos/ miR-193b-3p treatment (Fig. 5a). Interestingly, we found that $\mathrm{Bcl}-2$ expression was lower after $\mathrm{SAH}$ and was dramatically greater after Exos/miR-193b-3p treatment (Fig. 5b). The mRNA levels of $I L-1 \beta, I L-6$, and TNF- $\alpha$ were higher after SAH, and Exos/miR-193b-3p significantly reduced the expression of proinflammatory cytokines (Fig. $5 c-e$ ). Levels of IL-1 $\beta$, IL-6, and TNF- $\alpha$ in 

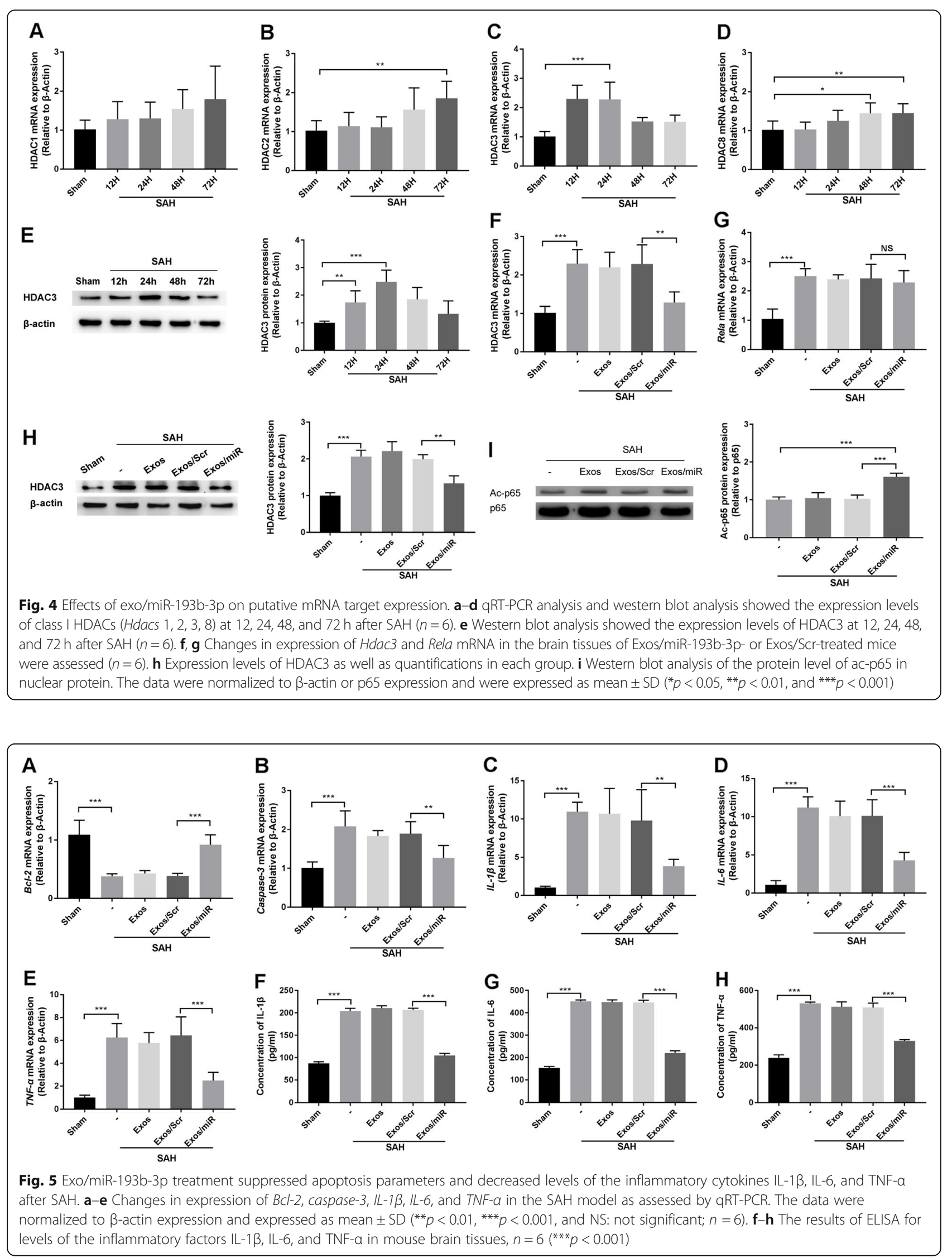
the cerebral hemisphere were measured using ELISA. We found that inflammatory cytokine levels were substantially higher with biphasic peaks after SAH. The data suggested that Exos/miR-193b-3p significantly lowered expression levels of IL-1 $\beta$, IL-6, and TNF- $\alpha$ in the brain after SAH (Fig. 5f-h).

\section{The effect of miR-193b-3p on neurological behavioral} impairment, brain edema, neurodegeneration, and BBB permeability in mice after $\mathrm{SAH}$

To determine the protective function of miR-193b-3p at $24 \mathrm{~h}$ post-SAH, neurological behavioral impairment, neurodegeneration, $\mathrm{BBB}$ permeability, and brain water content of mice in various groups were tested. As shown in Fig. 6a, the SAH group showed lower neurological scores than the sham group $(p<0.01)$. Exos/miR-193b$3 \mathrm{p}$ markedly improved neurological scores compared with the SAH and Exos/Scramble miRNA groups at $24 \mathrm{~h}$ post-SAH. Similarly, the degree of brain edema was significantly less pronounced in the Exos/miR-193b-3p group than in the $\mathrm{SAH}$ and Exos/Scramble miRNA groups (Fig. 6b). The numbers of FJC-positive cells were lower in the Exos/miR-193b-3p group and were higher in the SAH and Exos/Scramble miRNA groups compared with the sham group (Fig. 6c, d). SAH produced the amount of Evan's blue extravasation into the brain, which revealed the BBB injury $(p<0.001$, Fig. 6e). Also, enhanced Evan's blue extravasation was found in the Exos group and Exos/Scramble miRNA group as compared with the sham group. However, the BBB permeability was significantly attenuated by the Exos/miR$193 \mathrm{~b}-3 \mathrm{p}$ treatment $(p<0.001$, Fig. 6e).

\section{Discussion}

In the present study, firstly, we found that the circulating exosomal miRNA expression profiles showed distinct pattern differences between the SAH patients and healthy controls. Secondly, a SAH model of mice showed that plasma exosomal miR-193b-3p was greater than that in the sham controls, while it has lower expression in brain tissues. Thirdly, RVG-modified exosomes deliver miRNAs into the central nervous system (CNS) [11, 34], suggesting that miR-193b-3p delivery may be achieved using exosome encapsulation. Finally, we found that targeted delivery of miR-193b-3p into the brain after SAH reduced neuroinflammation and

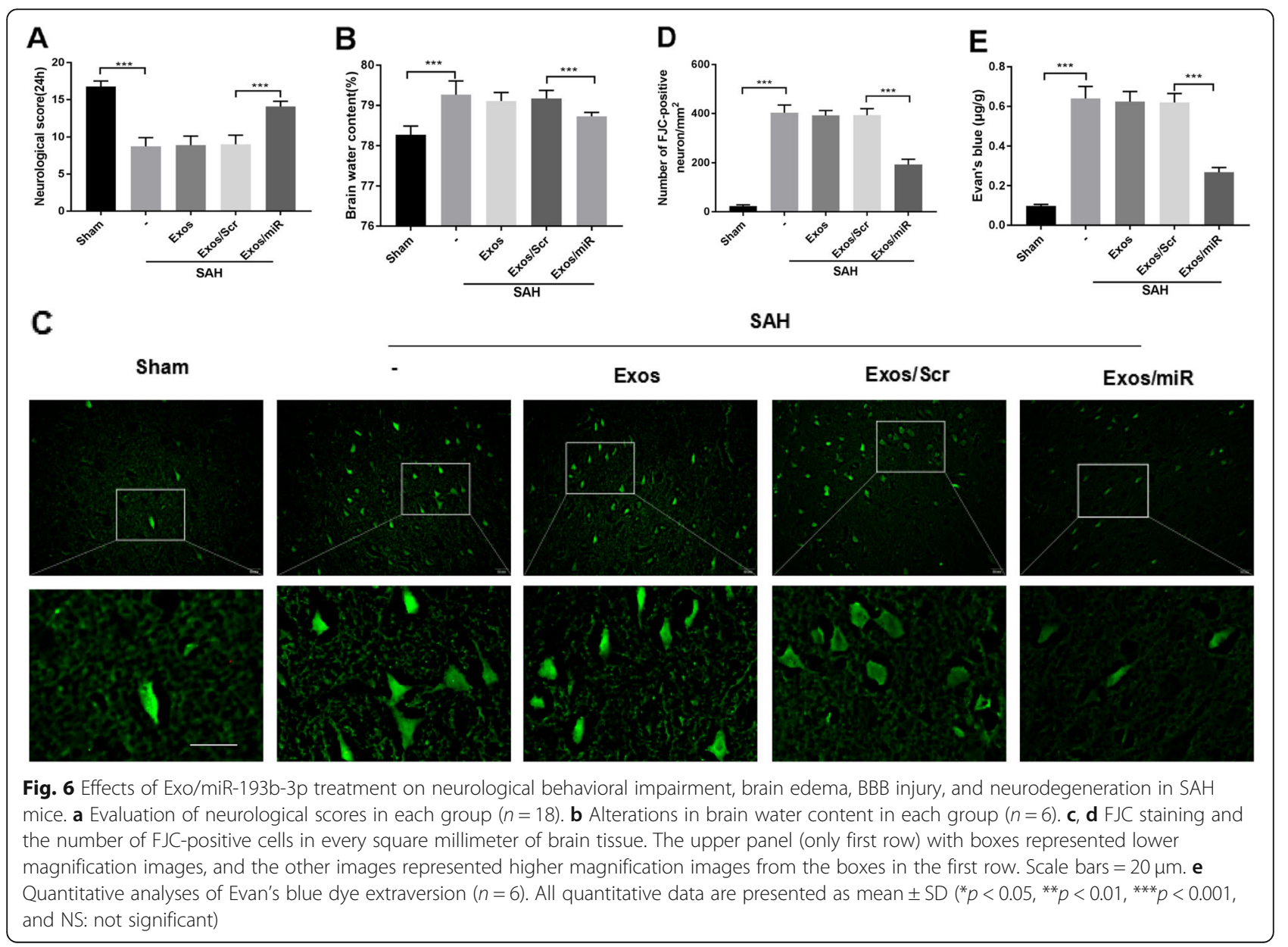


attenuated neuronal degeneration by inhibition of the HDAC3/NF-kB signal pathway.

We started with a total exosomal miRNA analysis using NGS to identify changes in expression levels of miRNAs between SAH patients and healthy controls. Subsequently, the NGS results for hsa-miR-486-3p, hsamiR-193b-3p, hsa-miR-369-3p, and hsa-miR-410-3p were verified using qRT-PCR experiments in a training set. We selected miR-193b-3p for follow-up validation to identify its role in a SAH mouse model. Exosomes can be enriched from peripheral blood samples released by brain cells and endothelial and peripheral cells. Exosomal miRNAs also can transport epigenetic information, affecting gene expression in cells distant to the cellular source $[22,35]$. Exosomes are a newly described mechanism of intercellular communication facilitating transfer of miRNAs, lipids, mRNAs, and proteins [36]. Importantly, exosome delivers miRNA or protein to the brain [25, 37]. Furthermore, modified exosomes with RVG fused to membrane glycoprotein Lamp2b efficiently delivered miRNA to the brain [34]. In our study, we found that FAM fluorescence was ferried to the brain of mice by exosomes. Originally, intravenously injection of DiI fluorescence ferried by RVG-Exos was used to achieve tailored delivery [11, 25, 34, 38]. RVG-exosomes delivered exogenous miR-124 to ischemic cortex and attenuated ischemic injury via cortical neurogenesis [11]. RVG-modified MSC-exosomes effectively balanced the inflammatory responses and improved learning and memory function in the brains of mice with Alzheimer's disease [25]. RVG-exosomes delivered $\alpha$-Syn siRNA to reduce alpha-synuclein aggregates in brains of mice with Parkinson's disease [38]. In this study, we also confirmed that RVG/Exos could successfully deliver miR-193b-3p to the brain using loaded exosomes with FAM-labeled scrambled miRNAs.

The inflammatory response occurred early after $\mathrm{SAH}$ and contributes to the progression of SAH-induced EBI $[5,6]$. Therapeutically, inhibition of neuroinflammation would be greatly beneficial to alleviate inflammationinduced EBI after SAH. Using this RVG/Exos/miR-193b$3 p$ in vivo treatment strategy, our data suggested that intravenous injection of miR-193b-3p loaded RVG/Exos causes significant decreases in HDAC3 mRNA and protein levels in brain tissues. Previous studies showed that HDAC3 was a class I histone deacetylase [39]. Histone deacetylase inhibition has a neuro-protective effect via reducing neuroinflammation [33], reported to be beneficial with respect to neurological functions in many diseases, including Alzheimer's disease [40], Huntington's disease [41], TBI, stroke [42], and spinal cord injury [33]. Recent studies reported that HDCA3 inhibition appears to suppress NF- $\mathrm{kB}$ transcriptional activity by maintaining the NF- $\mathrm{kB}$ p65 acetylated (inactive) state and restraining the inflammatory response [33, 43]. The NF- $k B$ signaling pathway occurs in passive post-injury necrosis and in damaged cells, activating microglia to secrete inflammatory cytokines and causing amplification of the inflammatory response cascade [44, 45]. In the present study, we found that levels of inflammatory cytokines (IL-1 $\beta$, IL- 6 , and TNF- $\alpha$ ) in brain tissue increased after SAH, associated with apoptosis (caspase-3, BCL-2), brain edema, and neurological defects. Inhibition of HDAC3 activity following miR-193b-3p treatment reduced expression levels of these inflammatory cytokines in vivo. NF- $\mathrm{KB}$ should be a transcription factor of inflammatory mediators that plays a key role in neuroinflammation [46, 47]. MiR-193b-3p significantly increased NF- $\mathrm{KB}$ p65 acetylation and reduced its transcriptional activity after SAH, suggesting that miR-193b-3p exerts neuroprotective effects. A previous study reported that miRNA-193b-3p inhibited hepatocyte apoptosis in selenium-deficient chickens [48]. Another study showed that HDAC3 deletion restrained neuroinflammation by regulating NF- $\mathrm{kB}$ p65 signaling, leading to neuroprotective effects after spinal cord injury. Previous studies also showed that HDAC3 inhibition had neuroprotective effects in stroke. HDAC3 inhibition increased the resistance of the brain to ischemic insult following middle cerebral artery occlusion (MCAO) [49]. HDAC3 inhibition exerted protective effects against diabetic stroke via the enhancement of oxidative stress, inhibition of apoptosis, and improvement of autophagy, all of which might be mediated by the upregulation of Bmal1 [50]. Furthermore, miR-494 in MCAO mouse model produced neuroprotective effects by reducing the expression levels of HDAC3 in neurons [51].

Although the present study aimed at investigating the tailored delivery of RVG-exosomes and potential mechanism of miR-193b-3p in neuroinflammation after SAH, there were a few methodological limitations. First, HDAC3 was a potential target of miR-193b-3p in brain tissues of mice after SAH, but it is not the only one. Second, miR-193b-3p attenuated neuroinflammation and led to neuroprotective effects after SAH. HDAC3 inhibitors acted on several cell types in the CNS, including neurons, astrocytes, and microglia [52]. miR-193b-3p and its target HDAC3 may also be regulated by other mechanisms, including autophagy or apoptosis $[49,51]$, possibly leading to neuroprotective effects. Further studies are required to account for the beneficial mechanisms of miR-193b-3p in vitro.

\section{Conclusions}

Taken together, our data suggested that intravenous injection of RVG-exosomes containing miR-193b-3p in a $\mathrm{SAH}$ mouse model promotes neuroprotective effects and anti-inflammatory responses. Inhibition of inflammation 
by miR-193b-3p affects the acetylation status of NF- $\mathrm{BB}$ p65 and reduces the expression of HDAC3. These findings suggest that RVG/Exos/miR-193b-3p may weaken neuroinflammation by suppressing the expression and the activity of HDAC3 and increasing NF- $\mathrm{kB}$ p 65 acetylation after SAH.

\section{Supplementary information}

Supplementary information accompanies this paper at https://doi.org/10. 1186/s12974-020-01745-0.

Additional file 1.

Additional file 2 .

\section{Abbreviations}

Ac-NF-KB: Acetylation of nuclear factor-kB; BBB: Blood-brain barrier; BCA: Bicinchoninic acid; BCL-2: B cell lymphoma-2; BMSCs: Bone marrow mesenchymal stem cells; BWC: Brain water content; CNS: Central nervous system; DCl: Delayed cerebral ischemia; DW: Dry weight; EBI: Early brain injury; ECL: Enhanced chemiluminescence; ELISA: Enzyme-linked immunosorbent assay; Exos: Exosomes; FJC: Fluoro-Jade C; HDAC3: Histone deacetylase 3; Lamp2b: Lysosome-associated membrane glycoprotein 2b; MiRNAs: MicroRNAs; NF-kB: Nuclear factor-kB; NGS: Next-generation sequencing; PBS: Phosphate buffer solution; PVDF: Polyvinylidene difluoride membranes; qRT-PCR: Quantitative real-time polymerase chain reaction; RVG: Rabies virus glycoprotein; SAH: Subarachnoid hemorrhage; SDSPAGE: Sodium dodecyl sulfate-polyacrylamide gels; TNF: Tumor necrosis factor; WW: Wet weight

\section{Acknowledgements}

Not applicable.

\section{Authors' contributions}

NL and DW conducted experiments and drafted the manuscript. TL, PP, GY, $\mathrm{XL}$, and $\mathrm{HL}$ contributed to the experiment design and statistical analysis. GC, $\mathrm{ZW}$, and HS designed the experiments and revised the manuscript. The authors read and approved the final manuscript.

\section{Funding}

This work was supported by National Key R\&D Program of China (no. 2018YFC1312600 and 2018YFC1312601), National Natural Science Foundation of China (no. 81701357), Anhui Province's Natural Science Foundation (no. 1708085QH181), Project of Jiangsu Provincial Medical Innovation Team (no. CXTDA2017003), Jiangsu Provincial Medical Youth Talent (no. QNRC2016728), Suzhou Key Medical Centre (no. Szzx201501), Scientific Department of Jiangsu Province (no. BE2017656), and Suzhou Government (LCZX201601).

\section{Availability of data and materials}

The datasets used and/or analyzed during the current study are available from the corresponding author on reasonable request.

\section{Ethics approval and consent to participate}

Study participants were recruited from the Department of Neurosurgery at The First Affiliated Hospital of Wannan Medical College, Wuhu City, China. The study was performed in accordance with the Declaration of Helsinki. Written informed consent was received from participants or valid proxies (family or a professional not directly involved in the study). All experiments were approved by the Ethics Committee of the First Affiliated Hospital of Soochow University and Research Ethics Committee of Wannan Medical College, and were performed according to the guidelines of the National Institutes of Health on the care and use of animals.

\section{Consent for publication}

Not applicable.

\section{Competing interests}

The authors declare that they have no competing interests.

\section{Author details}

'Department of Neurosurgery \& Brain and Nerve Research Laboratory, The First Affiliated Hospital of Soochow University, 188 Shizi Street, Suzhou 215006, Jiangsu Province, China. ${ }^{2}$ Department of Neurosurgery, First Affiliated Hospital of Wannan Medical College, 2 West Zheshan Road, Wuhu, Anhui Province, China. ${ }^{3}$ Non-coding RNA Research Center of Wannan Medical College, Wuhu, Anhui Province, China.

Received: 25 October 2019 Accepted: 13 February 2020

Published online: 25 February 2020

\section{References}

1. Vivancos J, Gilo F, Frutos R, Maestre J, Garcia-Pastor A, Quintana F, Roda JM, Ximenez-Carrillo A, Diez Tejedor E, Fuentes B, et al. Clinical management guidelines for subarachnoid haemorrhage. Diagnosis and treatment. Neurologia. 2014;29:353-70.

2. Lai NS, Zhang JQ, Qin FY, Sheng B, Fang XG, Li ZB. Serum microRNAs are non-invasive biomarkers for the presence and progression of subarachnoid haemorrhage. Biosci Rep. 2017;37:28.

3. Sheng B, Fang X, Liu C, Wu D, Xia D, Xu S, Lai N. Persistent high levels of miR-502-5p are associated with poor neurologic outcome in patients with aneurysmal subarachnoid hemorrhage. World Neurosurg. 2018;116:e92-9.

4. Helbok R, Schiefecker AJ, Beer R, Dietmann A, Antunes AP, Sohm F, Fischer M, Hackl WO, Rhomberg P, Lackner P, et al. Early brain injury after aneurysmal subarachnoid hemorrhage: a multimodal neuromonitoring study. Crit Care. 2015;19:75.

5. Dou Y, Shen H, Feng D, Li H, Tian X, Zhang J, Wang Z, Chen G. Tumor necrosis factor receptor-associated factor 6 participates in early brain injury after subarachnoid hemorrhage in rats through inhibiting autophagy and promoting oxidative stress. J Neurochem. 2017;142:478-92.

6. Liu W, Li R, Yin J, Guo S, Chen Y, Fan H, Li G, Li Z, Li X, Zhang X, et al. Mesenchymal stem cells alleviate the early brain injury of subarachnoid hemorrhage partly by suppression of Notch1-dependent neuroinflammation: involvement of Botch. J Neuroinflammation. 2019;16:8,

7. Lai NS, Wu DG, Fang XG, Lin YC, Chen SS, Li ZB, Xu SS. Serum microRNA210 as a potential noninvasive biomarker for the diagnosis and prognosis of glioma. Br J Cancer. 2015;112:1241-6.

8. Iranifar E, Seresht BM, Momeni F, Fadaei E, Mehr MH, Ebrahimi Z, Rahmati M, Kharazinejad E, Mirzaei H. Exosomes and microRNAs: new potential therapeutic candidates in Alzheimer disease therapy. J Cell Physiol. 2019;234:2296-305.

9. Roitbak T. Silencing a multifunctional microRNA is beneficial for stroke recovery. Front Mol Neurosci. 2018;11:58.

10. Ge X, Li W, Huang S, Yin Z, Yang M, Han Z, Chen F, Wang H, Lei P, Zhang $J N$. Increased miR-21-3p in injured brain microvascular endothelial cells following traumatic brain injury aggravates blood-brain barrier damage by promoting cellular apoptosis and inflammation through targeting MAT2B. J Neurotrauma. 2018. https://doi.org/10.1089/neu.2018.5728.

11. Yang J, Zhang $X$, Chen $X$, Wang L, Yang G. Exosome mediated delivery of miR-124 promotes neurogenesis after ischemia. Mol Ther Nucleic Acids. 2017:7:278-87.

12. Sun L, Zhang W, Li Z, Li M, Guo J, Wang H, Wang X. The expression of cerebrospinal fluid exosomal miR-630 plays an important role in the dysfunction of endothelial cells after subarachnoid hemorrhage. Sci Rep. 2019;9:019-48049.

13. Bache $S$, Rasmussen $R$, Rossing M, Laigaard FP, Nielsen FC, Moller K. MicroRNA changes in cerebrospinal fluid after subarachnoid hemorrhage. Stroke. 2017:48:2391-8.

14. Sheng B, Lai NS, Yao Y, Dong J, Li ZB, Zhao XT, Liu JQ, Li XQ, Fang XG. Early serum miR-1297 is an indicator of poor neurological outcome in patients with aSAH. Biosci Rep. 2018;38:BSR20180646.

15. Zhang L, Zhang S, Yao J, Lowery FJ, Zhang Q, Huang WC, Li P, Li M, Wang $X$, Zhang $C$, et al. Microenvironment-induced PTEN loss by exosomal microRNA primes brain metastasis outgrowth. Nature. 2015;527:100-4

16. Ramirez SH, Andrews AM, Paul D, Pachter JS. Extracellular vesicles: mediators and biomarkers of pathology along CNS barriers. Fluids Barriers CNS. 2018;15:018-0104.

17. Kanninen KM, Bister N, Koistinaho J, Malm T. Exosomes as new diagnostic tools in CNS diseases. Biochim Biophys Acta. 1862;2016:403-10.

18. Haney MJ, Klyachko NL, Zhao Y, Gupta R, Plotnikova EG, He Z, Patel T, Piroyan A, Sokolsky M, Kabanov AV, Batrakova EV. Exosomes as drug delivery vehicles for Parkinson's disease therapy. J Control Release. 2015;207:18-30. 
19. Kim DK, Nishida H, An SY, Shetty AK, Bartosh TJ, Prockop DJ. Chromatographically isolated CD63+CD81+ extracellular vesicles from mesenchymal stromal cells rescue cognitive impairments after TBI. Proc Natl Acad Sci U S A. 2016:113:170-5.

20. Lang FM, Hossain A, Gumin J, Momin EN, Shimizu Y, Ledbetter D, Shahar T, Yamashita S, Parker Kerrigan B, Fueyo J, et al. Mesenchymal stem cells as natural biofactories for exosomes carrying miR-124a in the treatment of gliomas. Neuro-Oncology. 2018;20:380-90.

21. Tian T, Zhang HX, He CP, Fan S, Zhu YL, Qi C, Huang NP, Xiao ZD, Lu ZH, Tannous BA, Gao J. Surface functionalized exosomes as targeted drug delivery vehicles for cerebral ischemia therapy. Biomaterials. 2018;150:137-49.

22. Chen J, Chopp M. Exosome therapy for stroke. Stroke. 2018;49:1083-90.

23. Fu C, Xiang Y, Li X, Fu A. Targeted transport of nanocarriers into brain for theranosis with rabies virus glycoprotein-derived peptide. Mater Sci Eng C Mater Biol Appl. 2018:87:155-66.

24. Deng W, Kandhi S, Zhang B, Huang A, Koller A, Sun D. Extravascular blood augments myogenic constriction of cerebral arterioles: implications for hemorrhage-induced vasospasm. J Am Heart Assoc. 2018;7:008623.

25. Cui GH, Guo HD, Li H, Zhai Y, Gong ZB, Wu J, Liu JS, Dong YR, Hou SX, Liu JR. RVG-modified exosomes derived from mesenchymal stem cells rescue memory deficits by regulating inflammatory responses in a mouse model of Alzheimer's disease. Immun Ageing. 2019;16:10.

26. Zou M, Huang W, Jiang W, Wu Y, Chen Q. Role of Cav-1 in HIV-1 Tatinduced dysfunction of tight junctions and Abeta-transferring proteins. Oxidative Med Cell Longev. 2019;14:3403206.

27. Garcia JH, Wagner S, Liu KF, Hu XJ. Neurological deficit and extent of neuronal necrosis attributable to middle cerebral artery occlusion in rats. Statistical validation. Stroke. 1995;26:627-34

28. Liu FY, Cai J, Wang C, Ruan W, Guan GP, Pan HZ, Li JR, Qian C, Chen JS, Wang $L$, Chen $G$. Fluoxetine attenuates neuroinflammation in early brain injury after subarachnoid hemorrhage: a possible role for the regulation of TLR4/MyD88/ NF-kappaB signaling pathway. J Neuroinflammation. 2018;15:347.

29. Meng F, Li Z, Zhang Z, Yang Z, Kang Y, Zhao X, Long D, Hu S, Gu M, He S, et al. MicroRNA-193b-3p regulates chondrogenesis and chondrocyte metabolism by targeting HDAC3. Theranostics. 2018;8:2862-83.

30. Kao MH, Lin TN. Histone deacetylases in stroke. Chin J Physiol. 2019;62:95-107.

31. San Jose-Eneriz E, Gimenez-Camino N, Agirre X, Prosper F. HDAC inhibitors in acute myeloid leukemia. Cancers. 2019;11.

32. Ziesche E, Kettner-Buhrow D, Weber A, Wittwer T, Jurida L, Soelch J, Muller $\mathrm{H}$, Newel $\mathrm{D}$, Kronich $\mathrm{P}$, Schneider $\mathrm{H}$, et al. The coactivator role of histone deacetylase 3 in IL-1-signaling involves deacetylation of p65 NF-kappaB. Nucleic Acids Res. 2013:41:90-109.

33. Chen S, Ye J, Chen X, Shi J, Wu W, Lin W, Li Y, Fu H, Li S. Valproic acid attenuates traumatic spinal cord injury-induced inflammation via STAT1 and NF-kappaB pathway dependent of HDAC3. J Neuroinflammation. 2018;15: 018-1193.

34. Alvarez-Erviti L, Seow Y, Yin H, Betts C, Lakhal S, Wood MJ. Delivery of siRNA to the mouse brain by systemic injection of targeted exosomes. Nat Biotechnol. 2011;29:341-5.

35. Casadei L, Calore F, Creighton CJ, Guescini M, Batte K, Iwenofu OH, Zewdu A, Braggio DA, Bill KL, Fadda $\mathrm{P}$, et al. Exosome-derived miR-25-3p and miR92a-3p stimulate liposarcoma progression. Cancer Res. 2017;77:3846-56.

36. Thery C, Witwer KW, Aikawa E, Alcaraz MJ, Anderson JD, Andriantsitohaina R, Antoniou A, Arab T, Archer F, Atkin-Smith GK, et al. Minimal information for studies of extracellular vesicles 2018 (MISEV2018): a position statement of the International Society for Extracellular Vesicles and update of the MISEV2014 guidelines. J Extracell Vesicles. 2018;7:1535750.

37. Shen H, Yao X, Li H, Li X, Zhang T, Sun Q, Ji C, Chen G. Role of exosomes derived from miR-133b modified MSCs in an experimental rat model of intracerebral hemorrhage. J Mol Neurosci. 2018;64:421-30.

38. Cooper JM, Wiklander PB, Nordin JZ, Al-Shawi R, Wood MJ, Vithlani M, Schapira AH, Simons JP, El-Andaloussi S, Alvarez-Erviti L. Systemic exosomal siRNA delivery reduced alpha-synuclein aggregates in brains of transgenic mice. Mov Disord. 2014;29:1476-85.

39. Reddy DS, Wu X, Golub VM, Dashwood WM, Dashwood RH. Measuring histone deacetylase inhibition in the brain. Curr Protoc Pharmacol. 2018;81:e41.

40. Cuadrado-Tejedor M, Perez-Gonzalez M, Garcia-Munoz C, Muruzabal D, Garcia-Barroso C, Rabal O, Segura V, Sanchez-Arias JA, Oyarzabal J, GarciaOsta A. Taking advantage of the selectivity of histone deacetylases and phosphodiesterase inhibitors to design better therapeutic strategies to treat Alzheimer's disease. Front Aging Neurosci. 2019;11:149.
41. Siebzehnrubl FA, Raber KA, Urbach YK, Schulze-Krebs A, Canneva F, Moceri S, Habermeyer J, Achoui D, Gupta B, Steindler DA, et al. Early postnatal behavioral, cellular, and molecular changes in models of Huntington disease are reversible by HDAC inhibition. Proc Natl Acad Sci U S A. 2018; 115:E8765-74.

42. Demyanenko S, Neginskaya M, Berezhnaya E. Expression of class I histone deacetylases in ipsilateral and contralateral hemispheres after the focal photothrombotic infarction in the mouse brain. Transl Stroke Res. 2018;9: 471-83.

43. McClure JJ, Inks ES, Zhang C, Peterson YK, Li J, Chundru K, Lee B, Buchanan A, Miao S, Chou CJ. Comparison of the deacylase and deacetylase activity of zinc-dependent HDACs. ACS Chem Biol. 2017;12:1644-55.

44. Pawlowska E, Szczepanska J, Wisniewski K, Tokarz P, Jaskolski DJ, Blasiak J. NF-kappaB-mediated inflammation in the pathogenesis of intracranial aneurysm and subarachnoid hemorrhage. Does autophagy play a role? Int J Mol Sci. 2018;19:E1245

45. You W, Zuo G, Shen H, Tian X, Li H, Zhu H, Yin J, Zhang T, Wang Z. Potential dual role of nuclear factor-kappa B in experimental subarachnoid hemorrhageinduced early brain injury in rabbits. Inflamm Res. 2016:65:975-84.

46. Xue YL, Zhang SX, Zheng CF, Li YF, Zhang LH, Hao YF, Wang S, Li XW. Silencing of STAT4 protects against autoimmune myocarditis by regulating Th1/Th2 immune response via inactivation of the NF-kappaB pathway in rats. Inflammation. 2019;8:019-00978.

47. Tian $M$, Yang $M, L i Z$, Wang $Y$, Chen $W$, Yang L, Li Y, Yuan H. Fluoxetine suppresses inflammatory reaction in microglia under OGD/R challenge via modulation of NF-kappaB signaling. Biosci Rep. 2019;39:30.

48. Liu T, Yang T, Xu Z, Tan S, Pan T, Wan N, Li S. MicroRNA-193b-3p regulates hepatocyte apoptosis in selenium-deficient broilers by targeting MAML1. J Inorg Biochem. 2018;186:235-45.

49. Yang X, Wu Q, Zhang L, Feng L. Inhibition of histone deacetylase 3 (HDAC3) mediates ischemic preconditioning and protects cortical neurons against ischemia in rats. Front Mol Neurosci. 2016;9:131.

50. Zhao B, Yuan Q, Hou JB, Xia ZY, Zhan LY, Li M, Jiang M, Gao WW, Liu L. Inhibition of HDAC3 ameliorates cerebral ischemia reperfusion injury in diabetic mice in vivo and in vitro. J Diabetes Res. 2019;2019:8520856.

51. Zhao H, Li G, Zhang S, Li F, Wang R, Tao Z, Ma Q, Han Z, Yan F, Fan J, et al. Inhibition of histone deacetylase 3 by MiR-494 alleviates neuronal loss and improves neurological recovery in experimental stroke. J Cereb Blood Flow Metab. 2019;39:2392-405.

52. Langley B, Brochier C, Rivieccio MA. Targeting histone deacetylases as a multifaceted approach to treat the diverse outcomes of stroke. Stroke. 2009; 40:2899-905.

\section{Publisher's Note}

Springer Nature remains neutral with regard to jurisdictional claims in published maps and institutional affiliations.

\section{Ready to submit your research? Choose BMC and benefit from:}

- fast, convenient online submission

- thorough peer review by experienced researchers in your field

- rapid publication on acceptance

- support for research data, including large and complex data types

- gold Open Access which fosters wider collaboration and increased citations

- maximum visibility for your research: over $100 \mathrm{M}$ website views per year

At $\mathrm{BMC}$, research is always in progress.

Learn more biomedcentral.com/submissions 